\title{
Robot Pose Measurement Based on Sensor Fusion
}

\author{
Jiwu Wang ${ }^{1}$ \\ School of Mechanical, Electronic and Control Engineering, Beijing Jiaotong University \\ Beijing 100044, China ${ }^{1}$ \\ Shunkai Zheng', Fangbo Liao ${ }^{1}$ \\ School of Mechanical, Electronic and Control Engineering, Beijing Jiaotong University \\ Beijing 100044, China ${ }^{1}$ \\ Masanori Sugisaka ${ }^{2}$ \\ Alife Robotics Corporation Ltd, Japan and Open University, United Kingdom ${ }^{2}$ \\ E-mail: jwwang@bjtu.edu.cn; sugisaka3@mocha.ocn.ne.jp
}

\begin{abstract}
In the motion control research of mobile robot, the mobile robot pose is obtained by derivation of the Kinematic model of the robot. Generally, the motion model relies simply on the data from encoder feedback. Due to cumulative error, the robot pose accuracy is relatively poor by mileage positioning with encoder. Here a new method is put forward based on data fusion of gyro sensor with the encoder data, and the robot pose accuracy is analyzed and improved.
\end{abstract}

Keywords: sensor fusion; DCM; EKF filtering

\section{Introduction (Basic Measurement Unit)}

The idea of pose measurement system based on the fusion is as following: firstly, build motion analysis model; then with the inertial sensor data, give the accurate pose estimation.

Hardware IMU measurement system used in this paper to achieve mini atomization of inertial measurement through the MEMS technology, the IMU module contains ITG3205 gyroscope, ADXL345 accelerometer, HMC5883L electronic compass. The sensor is connected to the microprocessor via a bus, and the microprocessor reads and writes data correction of sensors. ${ }^{1}$
Gyroscope, accelerometer, compass data from these three sensors output are implemented on an I ${ }^{2} \mathrm{C}$ bus interface, the sensor run as a slave device, a microprocessor done as the master device. ${ }^{2}$ The system structuer is shown in Figure 1.

The MEMS sensor with 3 axes digital output angular velocity, each axis is provided with a 16bit $\mathrm{A} / \mathrm{D}$ converter, built-in low-pass filter with adjustable bandwidth, shown in figure 2 . $^{3}$ The measuring range is $\pm 2000^{\circ} / \mathrm{s}$, and ADC output frequency can be adjust in the range of $8000 \mathrm{HZ}$ to $3.9 \mathrm{HZ}$. 


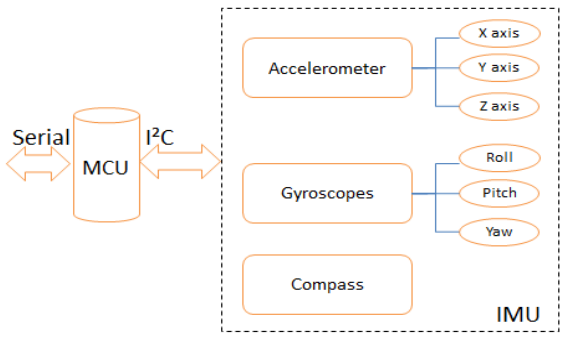

Fig. 1 IMU pose measurement system architecture diagram

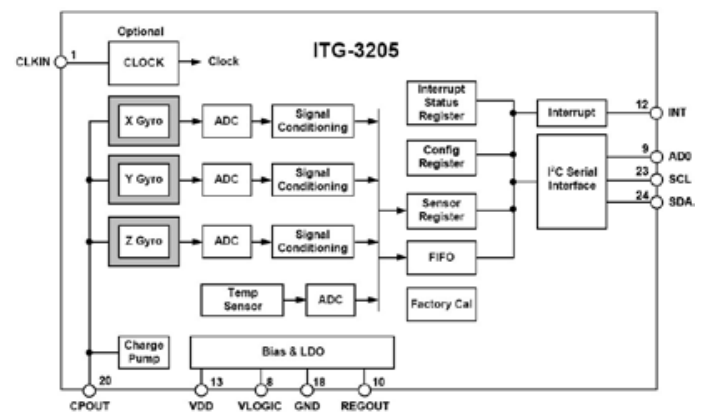

Fig. 2 ITG-3205 structure diagram

ADXL345, the three axis acceleration of an ultra low power design, measurement range of up to $\pm 16 \mathrm{~g}$. Moreover, the device also has the knock detection, free fall detection, etc., shown in figure 3 .

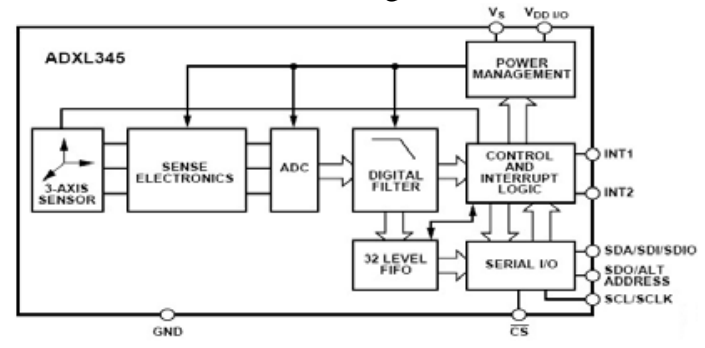

Fig. 3 ADXL345 structure diagram

Electronic compass HMC5885, as a low cost electronic compass with a high precision, has a 12 bit ADC conversion accuracy, compass navigation precision can be $1^{\circ}$ or $2^{\circ}$.

\section{Analysis of DCM Algorithm}

The DCM algorithm is as followings: a nonlinear correlation gyro measurement data and the direction cosine change rate of difference equations. ${ }^{4}$ The flow algorithm in digital system is shown in figure 4 .

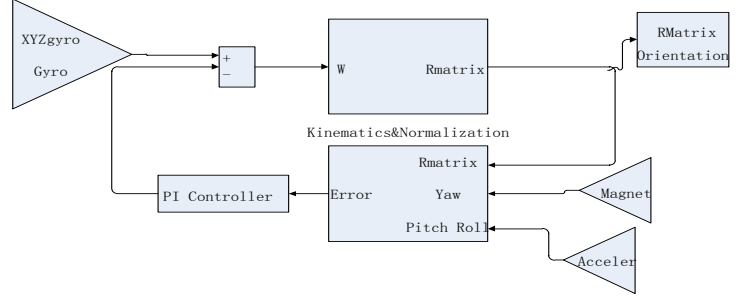

Fig.4 ITG-3205 structure diagram

\subsection{The kinematics analysis algorithm}

The kinematics equation is

$$
\frac{d r(t)}{d t}=\omega(t) \times r(t)
$$

$\omega(t)$ is the angular velocity vector.

With the initial state and rotating vector, it can be:

$$
\left\{\begin{array}{c}
r(t)=r(0)+\int_{0}^{t} d \theta(\tau) \times r(\tau) \\
d \theta(\tau)=\omega(\tau) d \tau
\end{array}\right.
$$

Where $r(0)$ is the initial vector.

Furtherly, in the global coordinate system, using the method of approximate matrix, it will be

$$
\left\{\begin{array}{c}
r_{\text {global }}(t+d t)=r_{\text {global }}(t)+r_{\text {global }}(t) \times d \theta(t) \\
d \theta(t)=\omega(t) d t
\end{array}\right.
$$

For error compensation of angular velocity, a modified angular velocity is introduced, which is determined by the angular velocity and its correction.

$$
\omega(t)=\omega_{\text {gyro }}(t)+\omega_{\text {cor }}(t)
$$

Formula (5) is the DCM update equation matrix about gyroscope.

$$
R(t+d t)=R(t)\left[\begin{array}{ccc}
1 & -d \theta_{z} & d \theta_{y} \\
d \theta_{z} & 1 & -d \theta_{x} \\
-d \theta_{y} & d \theta_{x} & 1
\end{array}\right]
$$

Here, $d \theta_{x}=\omega_{x} d t, d \theta_{y}=\omega_{y} d t, d \theta_{z}=\omega_{z} d t$

\subsection{Drift compensation}

In order to calculate the Roll, Pitch, Yaw for compensation, a feedback PI controller is introduced. The controller error calculation of Roll and Pitch uses the acceleration and the electronic compass Yaw error. In our wxperiment, the following figure is drawn based on the sensor data by IMU sensor. 


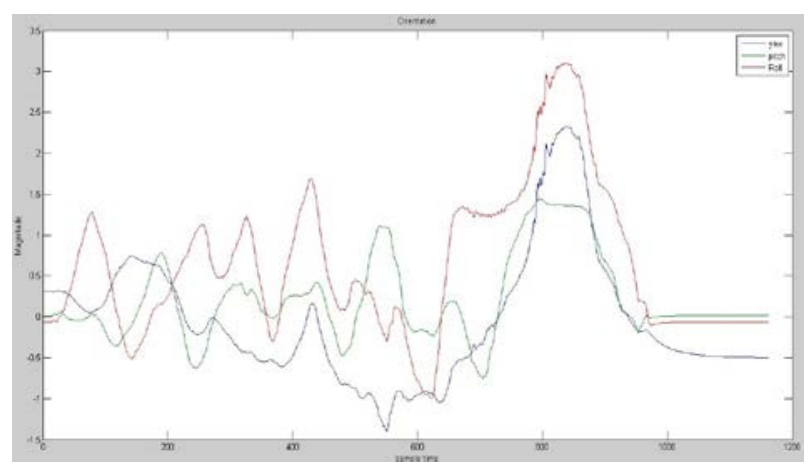

Fig. 5. Orientation sampling curve

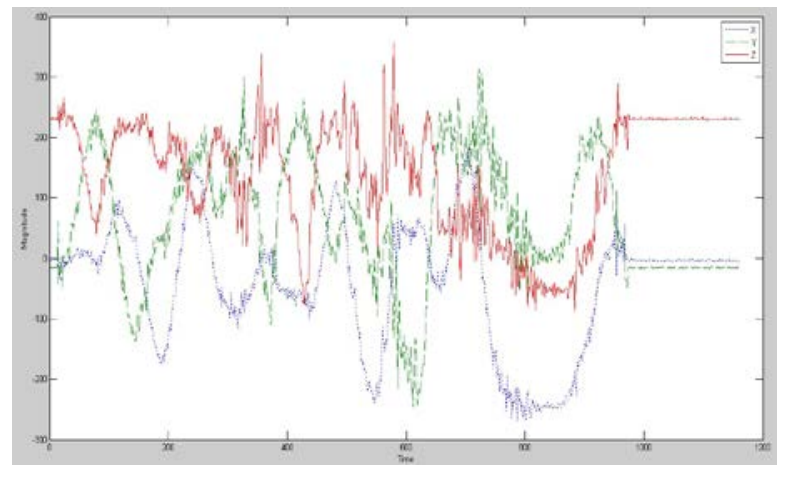

Fig. 6. Three axis speed curves

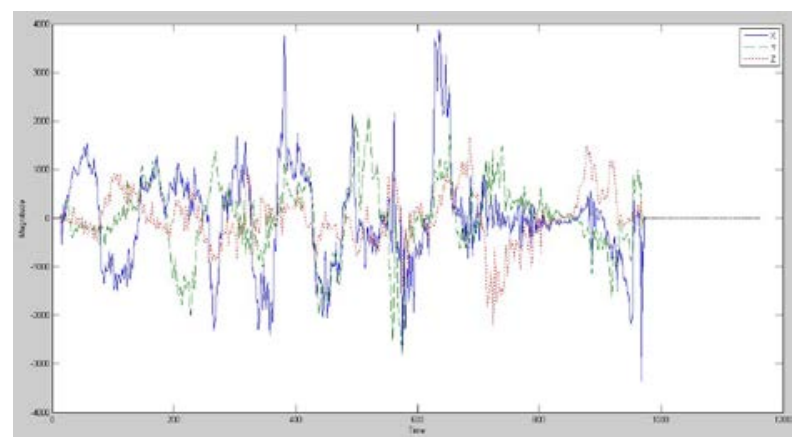

Fig. 7. Three axis angle velocity curves

In figure 6, the movement state of three axises are given. $\mathrm{Z}$ axis acceleration is caused by the acceleration of gravity. In figure 7 , they are three axis angular velocity.

\section{The Extended Kalman Filter Algorithm}

Now the encoder measuring odometry data (Odom) and the inertial measurement data (IMU) can be determined. The full state vector in the space is expressed as $x_{k}=\left[X_{k}, Y_{k}, Z_{k}, \phi_{k}, \theta_{k}, \psi_{k}, v_{k}, \omega_{k}\right]^{T}$
With $\boldsymbol{\Delta} \boldsymbol{t}$ time, we can get

$$
\left\{\begin{array}{c}
v_{R}=\eta \frac{N_{L}}{\Delta t} \\
v_{R}=\eta \frac{N_{R}}{\Delta t} \\
\omega=\frac{v_{R}-v_{L}}{L} \\
v=\omega * L
\end{array}\right.
$$

Where $\boldsymbol{\eta}$ is parameter of the wheel diameter and encoder. The pose after $\boldsymbol{\Delta} \boldsymbol{t}$ will be

$$
x_{k+1}=\left(\begin{array}{l}
X_{k}+v_{k} \cos \left(\psi_{k}\right) \Delta t \\
Y_{k}+v_{k} \sin \left(\psi_{k}\right) \Delta t \\
0 \\
0 \\
0 \\
\psi_{k}+\omega_{k} \Delta t \\
v_{k} \\
\omega_{k}
\end{array}\right)
$$

The EKF data integration system model is given as follows:

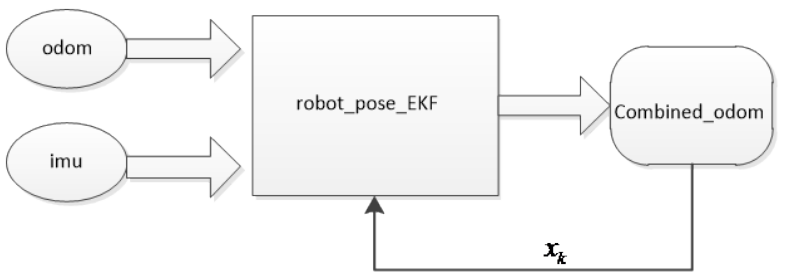

Fig. 8. EKF data integration system model

The extended Kalman filter with fusion of encoder and gyroscope data, the system state equation is derived:

$$
\boldsymbol{x}_{k+1}=\phi_{k+1 \mid k} \boldsymbol{x}_{k}+\Gamma_{k} W_{k}
$$

The measurement model for encoder is

$$
y_{o d o m, k}=C_{o d o m} x_{k}=\left[\begin{array}{c}
X_{k} \\
Y_{k} \\
\psi_{k} \\
v_{k} \\
\omega_{k}
\end{array}\right]+e_{o d o m, k}(d)
$$

The simplified IMU mathematical model is 


$$
y_{\text {gyro }, k}=C_{g y r o} x_{k}=\left[\begin{array}{l}
\psi_{k} \\
\omega_{k}
\end{array}\right]+e_{g y r o, k}
$$

Error covariance of the matrix is:

$$
R(d)=\left[\begin{array}{cc}
R_{\text {odom }}(d) & 0 \\
0 & R_{\text {gyro }}
\end{array}\right]
$$

With above the measurement model and state transition model, using the extended Kalman filtering, the robot pose estimation can be determined.

In order to validate the Kalman filter, with the above data, matlab simulation is performed.

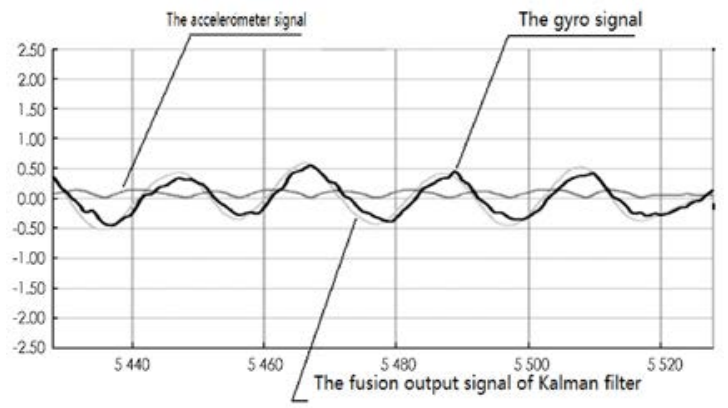

Fig. 9. The introduction of Kalman filter wave curve

Figure 9 is the introduction of Kalman filter wave curve. The output curve gets smoothing, and angle estimation accuracy is greatly improved.

\section{Conclusions}

In this paper, the Kalman filtering accelerometer, gyro and the electronic compass fusion method is studied. Based on dynamic test data, the Kalman filter compensates the sensor drift, noise and other factors caused by the accelerometer, gyroscope and the electronic compass. It reduces the angle measurement error, and improves the operation accuracy. The experiment results show the effectiveness of the proposed method.

\section{References}

1. R. A. Mohammad, Sensor fusion by pseudo information measure: a mobile robot application, ISA Transactions, 41 ( 2002)283-301.

2. A. J. Terrya, M. Z. John, Sensor fusion by a novel algorithm for time delay estimation, Digital Signal Processing, 22(2012) 439-452.

3. R. S. Blum, Multisensor image fusion performance limits from an estimation theory perspective, Information Fusion, 7( 2006) 250-263.
4. A. Weckenmann, X. Jiang, Multisensor data fusion in dimensional metrology, CIRP Annals-Manufacturing Technology, 58(2009)701-721.

5. H. Youngjoon, H. Hernsoo, Localization and classification of target surfaces using two pairs of ultrasonic sensors, Robotics and Autonomous Systems, 33(2000) 31-41.

6. J. B. Gao, C. J. Harris, Some remarks on Kalman filters for the multisensor fusion, Information Fusion, 3(2002)191-201

7. G. Gerasimos, G. Rigatos. Extended kalman and particle filtering for sensor fusion in motion control of mobile robots, Mathematics and Computers in Simulation, 81(2010)590-607 